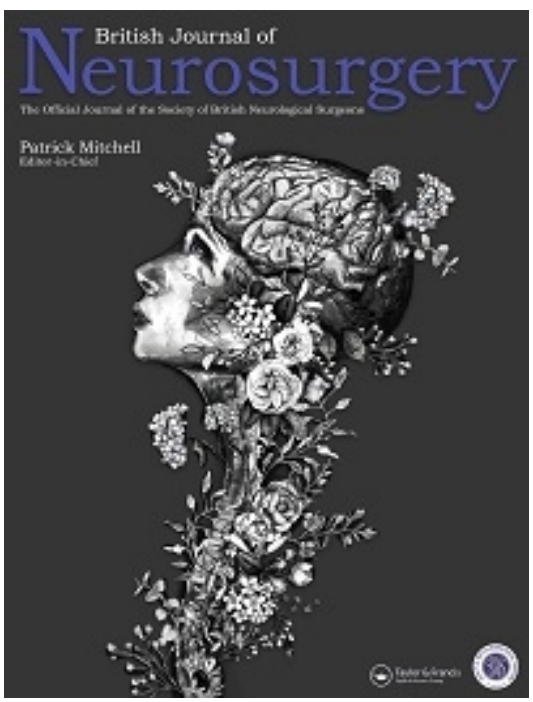

\title{
Comparison between outcomes of endovascular and surgical treatments of Ruptured Anterior Communicating Artery Aneurysms
}

\begin{tabular}{|r|l|}
\hline Journal: & British Journal of Neurosurgery \\
\hline Manuscript ID & CBJN-2019-0417.R2 \\
\hline Manuscript Type: & Original Article \\
\hline Author: & 10 -Jun-2020 \\
\hline & $\begin{array}{l}\text { Harris, Lauren; Queen's Hospital, } \\
\text { Hill, Ciaran; Queen's Hospital } \\
\text { Elliot, Matthew ; Queen's Hospital } \\
\text { Fitzpatrick, Teresa; Queen's Hospital } \\
\text { Ghosh, Anthony; Queen's Hospital } \\
\text { Vindlacheruvu, Raghu; Queen's Hospital }\end{array}$ \\
\hline Keywords: & $\begin{array}{l}\text { Aneurysm, Clipping, Coiling, Recurrence, Surgery, Anterior } \\
\text { Communicating Artery Aneurysm }\end{array}$ \\
\hline
\end{tabular}

\section{SCHOLARONE" Manuscripts}




\title{
Comparison between outcomes of endovascular and surgical treatments of Ruptured
}

\section{$\underline{\text { Anterior Communicating Artery Aneurysms }}$}

Authors:

Lauren Harris, Ciaran Scott Hill, Matthew Elliot, Teresa Fitzpatrick, Anthony Ghosh, Raghu

Vindlacheruvu

Affiliations: Barking, Havering and Redbridge University Hospital NHS Trust, Queen's Hospital, Romford

Corresponding Author:

Lauren Harris

Queen's Hospital, Rom Valley Way, Romford, RM70AG

Lauren.harris7@nhs.net

Presentation at conference:

BNVG (British Neurovascular Group Meeting) February 2019

Word Count - 2961

\author{
Abbreviations and acronyms: \\ ACOM: Anterior communicating artery \\ SAH: Subarachnoid haemorrhage
}


ISAT: International Subarachnoid Aneurysm Trial

CTA: CT angiography

DSA: Digital Subtraction Angiography

MRA: Magnetic Resonance Angiography

WFNS: World Federation of Neurological Surgeons

GOS: Glasgow Outcome Scale

CNS: Clinical nurse specialist

NCEPOD: National confidential enquiry into patient outcome and death

MDT: Multidisciplinary team 


\section{Abstract}

\section{Introduction}

The natural history and optimal treatment of previously ruptured anterior communicating artery (ACOM) aneurysms that recur is unknown. This study looks at rates of complications and recurrences of ruptured ACOM aneurysms treated endovascularly and surgically.

\section{Materials and methods}

A retrospective observational study of all patients presenting to a single tertiary neurosurgical centre with the first presentation of subarachnoid haemorrhage (SAH) secondary to a ruptured ACOM aneurysm. Data was collected from November 2012 to September 2018 and included baseline demographics, aneurysm characteristics, management, complications, follow-up imaging, and clinical outcomes.

\section{Results}

137 patients were included in the study. 113 aneurysms were coiled and 19 were clipped. Management decisions were taken by the multidisciplinary team based on aneurysm morphology or the presence of a haematoma exerting mass effect. There were 187.5 patientyears of follow-up, with a median of 3 years (range 0-73 months). Rates of vasospasm, infarction, CSF diversion, rebleed, length of stay, and functional outcome were not significantly different in the two cohorts. There was a statistically significant increase in the risk of ACOM recurrence in the coiled group when compared to the clipped group at one year $(p=0.0433) .15$ patients required further treatment at a median time of 16 months. In a 
subgroup group analysis of coiled aneurysms, there was no statistical differences in rates of rebleeding or the functional outcome in those that had aneurysm recurrence and those that did not.

\section{Conclusions}

This study suggests patients with aneurysms treated by endovascular coiling have an increased risk of recurrence versus those treated with clipping. However, the risk of rebleed was not statistically significant. The prevention and impact of recurrence and residual aneurysms remains incompletely understood. Hence, treatment decisions should be taken by patients after they have been given carefully considered recommendations from the multi-disciplinary team.

\section{Keywords}

Anterior communicating artery (ACOM) aneurysm

Clipping

Surgery

Endovascular coiling

Recurrence 


\section{Introduction}

Intracranial aneurysm rupture causing subarachnoid haemorrhage (SAH) occurs with a frequency of 6-8/100,000 in the developed world (Molyneux et al. 2002). Anterior communicating artery (ACOM) aneurysms are the most common and account for approximately 30\% of aneurysmal SAH in adults (Molyneux et al. 2002, Kirkpatrick et al. 2006, Fang et al. 2014). Aneurysms in this location can be technically challenging from a surgical perspective. Since the introduction of Guglielmi Detachable Coils in 1991, the inherently less invasive endovascular approach has become a feasible treatment option (Molyneux et al. 2002, Fang et al. 2014).

The surgical approach to ACOM aneurysms in the interhemispheric fissure can be challenging because of arterial relationships, complex regional flow dynamics, posterior fundus projections, barriers presented by the diencephalon and recent haemorrhage (Proust et al. 2003, Fang et al. 2014). ACOM artery anomalies occur in up to $35 \%$ of cases (Li et al. 2013). Endovascular treatment for ACOM aneurysms also has difficulties. They can be technically demanding if their size is $3 \mathrm{~mm}$ or less, if the aneurysm is complex and incorporating ACOM arteries, if the fundus projects posteriorly, if there is a wide neck, or when access is restricted by acute vessel angles (Proust et al. 2003, Finitsis et al. 2010, Fang et al. 2014).

The International Subarachnoid Aneurysm Trial (ISAT) and other studies have shown patients with ruptured aneurysms may have significant advantages in disability independent survival and functional outcomes when treated endovascularly versus surgically (Molyneux et al. 2002, 2009, 2015, Finitsis et al. 2010, McDougall et al. 2012, Lindgren et al. 2018). Reported 
disadvantages of endovascular treatment include rebleeding and recurrence, and questions remain about the durability of coils over time (Molyneux et al. 2002, 2009, 2015, CARAT Investigators 2006, Mitchell et al. 2008, Li et al. 2013, Fang et al. 2014, Spetzler et al. 2015).

\begin{abstract}
A limited number of studies have detailed the clinical outcomes and complications specific to ACOM aneurysms, with fewer focusing on ruptured aneurysms (Fang et al. 2014). The natural history and optimal treatment of previously ruptured ACOM aneurysms that recur is unclear. We aim to identify the immediate and long-term outcomes of endovascular treatment versus open surgery, including complications, recurrences and rebleeding.
\end{abstract}

\title{
Materials and methods
}

All consecutive patients presenting to a single tertiary neurosurgical centre in the United Kingdom with a first presentation of a SAH secondary to a ruptured ACOM aneurysm from November 2012 to September 2018 were included in this study. The diagnosis was based on computerised tomographic angiography (CTA) and digital subtraction angiography (DSA). Cases were reviewed by three interventional neuroradiologists and two consultant neurovascular surgeons and diagnosis and management decided by multidisciplinary team (MDT) consensus. Exclusion criteria included non-ACOM aneurysm SAH or second presentation. All data was collected retrospectively.

\footnotetext{
Baseline data collected included patient demographics, World Federation of Neurological Surgeons (WFNS) grade, Fischer grade and aneurysm characteristics. Incidence of short- and long-term complications were assessed from medical records, follow-up imaging and clinical letters. These included residual filling, vasospasm, hydrocephalus, seizure, recurrence, rebleed
} 
and mortality. Outcomes were assessed using the Glasgow Outcome Scale (GOS). All patients were clinically evaluated before hospital discharge and informally in outpatient clinic followup. Cognitive and memory deficits were identified by the therapy teams at discharge and formally assessed on neuropsychology follow-up. Data on residual or recurrent aneurysms was extracted from imaging reports by consultant neuroradiologists. If the information was not readily available, a further imaging review was done by the MDT to reduce intra-observer variability.

For the analysis the population was divided into two cohorts: patients who were clipped versus patients who were coiled. Subgroup analysis was performed on the coiled patients, comparing those who had a recurrence to those who did not. Statistical analysis of the results was made using chi-squared test and student $t$ test.

\section{Results}

137 patients with a SAH from an ACOM aneurysms presented between November 2012 and September 2018. 80 (58.4\%) were female and 57 (41.6\%) were male, with a mean age of 58.1 +/- 15.0 years and range of 19 to 92 years. 113 were treated endovascularly (82.5\%), 19 surgically $(13.9 \%)$, with the remaining five patients $(3.6 \%)$ were deemed not fit for intervention (Figure 1). There were 187.5 patient-years of follow-up, with a median of 3 years (range 0-73 months).

\section{Baseline characteristics}


The baseline characteristics of the two cohorts are shown in Table 1. The two groups were well matched for age, sex and comorbidities. There was a higher frequency of smokers in the coiled cohort. Two cases in the surgically treated group were in patients with known ACOM aneurysms under surveillance with a plan to treat if required, versus none in the endovascular group $(\mathrm{p}=0.0005)$. The aneurysm characteristics were well matched in both cohorts (Table 2$)$, including size of aneurysm neck, dome, dome to neck ratio, maximum diameter, aneurysm size, parent vessel diameter, flow angle, size ratio, number of lobes and fundus projection. The aneurysm incorporated the ACOM artery more frequently in the clipped cohort (47\% versus $23 \%, \mathrm{p}=0.0303)$.

The 'good grade' WFNS score, and good motor score (M6 - obeying commands), favoured endovascular treatment whereas the grade IV WFNS, and poor motor score (M1 - no motor response), tended to be treated surgically ( $p=0.0046$ and $p=0.0005$ respectively).

\section{Treatment}

Indications for clipping included aneurysm morphology (for example wide neck or very small) in 13 cases $(68.4 \%)$, haematoma exerting mass effect in two cases $(10.5 \%)$, out of hours in two $(10.5 \%)$ and multifactorial in two (10.5\%). Of the 19 patients who had surgical clipping, 14 (73.7\%) had DSA pre-operatively. Seven (50\%) of these had a failed attempt at coiling, four (57.1\%) failed due to a wide neck, one (14.3\%) due to a migrated coil, one $(14.3 \%)$ due to internal carotid artery stenosis and one (14.3\%) for tortuous vessel anatomy. For all 19 patients in the surgical cohort, intraoperative indocyanine green angiography and Doppler ultrasonography was used. Of the 113 patients treated endovascularly, three patients required a stent on initial treatment, and none required a flow diverter. 
The time to treat was equivalent in the two treatment arms, both with a median of one day. 4 (21\%) clipped patients, and $14(12 \%)$ coiled patients were treated more than 48 hours after presentation to the unit.

Complete obliteration (no filling of contrast medium in the dome, body or neck) was achieved in $94.7 \%$ of the clipped patients (one patient had a residual) and $80.5 \%$ in the coiled group. 22 of the coiled cohort had a residual, $18(81.8 \%)$ were Raymond Roy classification II, and four (18.2\%) were Raymond Roy classification IIIb, assessed on immediate peri-procedural DSA. No patients had a planned two-stage approach. For the clipped cohort, imaging modality depended on anatomical and patient specific considerations and included DSA (53.8\%), CTA $(30.8 \%)$ or MRA $(15.4 \%)$.

\section{Complications}

In the coiled cohort, three patients $(2.7 \%)$ had peri-procedural complications, including one aneurysm rupture $(0.9 \%)$, and two thromboembolic events $(1.8 \%)$, resulting in transient neurological deficits (Table 3). There was no statistically significant difference in the rates of seizures, vasospasm or infarcts between the two treatment groups. Nine patients $(47.4 \%)$ in the clipped cohort and $29(25.7 \%)$ in the coiled cohort suffered from symptomatic vasospasm at a median of five days post admission. Of these, seven (77.8\%) clipped and 21 (72.4\%) coiled patients required chemical or mechanical angioplasty. Indication to treat was the presence of new neurological symptoms refractory to optimal medical management in an intensive care setting (including induced hypertension). Restitution of the calibre of the vessel was obtained in all angioplasty cases, as determined by intra-operative angiography, and no angioplasty 
related complications occurred. Eight surgical (42.1\%) and 27 endovascular (23.9\%) patients had radiological evidence of infarcts.

There was no statistical difference in the rate of CSF diversion or shunts in the two cohorts. Nine (47.4\%) of the clipping cohort had an external ventricular drain, and $30(26.5 \%)$ of the coiled cohort had a CSF diversion procedure (21 external ventricular drains, 3 daily lumbar punctures, 6 lumbar drains).

\section{Outcomes}

There was no statistically significant difference in the length of hospital stay, discharge destination or mortality between the two groups. Mortality rate was $21.1 \%$ in the clipping cohort and $13.7 \%$ in the coiled cohort, at a median of day 28 and 11.5 respectively. This includes deaths related directly to the SAH and its complications. There was no difference in the two cohorts on stratifying mortality by initial grade of presentation $(100 \%$ of the clipped cohort and $66.7 \%$ of the coiled cohort who died presented with poor grade WFNS III-V, p = $0.2593)$

All patients were clinically evaluated before hospital discharge and on clinic follow-up. There was no statistical difference in functional outcome between groups, with a favourable outcome (GOS 4 or 5 ) in $57.9 \%$ of the clipped cohort and $78.4 \%$ of the coiled. Cognitive and memory deficits were seen in $5.3 \%$ in the clipped group and $6.2 \%$ in the coiled.

\section{Follow-up}


Follow-up included imaging, either formal DSA or 3D Time of Flight MRA, clinic visits and MDT discussions. $46.7 \%$ of the clipped cohort had further imaging (MRA (42.9\%), CTA $(42.9 \%)$ or DSA (14.3\%)), and patients with no residual were discharged after one year. Coiled patients had follow-up (usually in the form of MRA) at six months, one year, three years and five years, and thereafter they were discharged if stable (Figure 2).

There was a statistically significant increase in the risk of ACOM recurrence in the coiled group when compared to the clipped group at one year $(\mathrm{p}=0.0433)$. Recurrences ranged from immediately post treatment up to 29 months (Figure 3). 15 aneurysms that recurred after coiling were re-treated, 14 endovascularly (including coils, flow diverter or web as appropriate) and one surgically, at a median time of 16 months. $10.6 \%$ of the initial cohort of coiled patients had a second procedure. No patients have yet had a third procedure although follow-up continues. Recurrence over time for the coiled cohort can be seen in Figure 4. One patient had a late rebleed.

Subgroup analysis of coiled aneurysms that had a recurrence versus those that did not showed that younger age $(p=0.0466)$ and larger aneurysm size $(p=0.0039)$ were associated with recurrence. There was no difference with sex or smoking status. There was no difference in rebleeding rate or functional outcomes between the two groups (Table 4).

The rate of rebleeding from the ACOM aneurysm following coiling was $5.3 \%$ versus $0 \%$ for the clipped cohort. Five (4.4\%) rebled early (less than 30 days from ictus), of whom three had completely occluded aneurysms on post-treatment imaging (two of these patients did not survive) and two had residual necks (Table 5). Only one patient ( $0.9 \%)$ had a late rebleed on 
day 433; this was from an aneurysm recurrence which was not picked up earlier as he did not attend his follow-up.

\section{Discussion}

At our institution, all SAH patients are discussed at an MDT meeting. Endovascular treatment is commonly the intervention of choice for ruptured ACOM aneurysms. If not suitable for endovascular treatment, if there was an unsuccessful coiling attempt, or following MDT decision, patients may be offered microsurgical clipping. In accordance with the NCEPOD 'Managing the flow' national guidelines, we aim to treat all ruptured aneurysm within 48 hours of rupture (Alleway et al. 2013). In general, we do not advocate the placement of stents to treat patients presenting with acute aneurysm rupture, unless specific MDT rationale.

Our unit consists of two consultant neurovascular neurosurgeons with 17 years combined experience, and three interventional neuroradiologists with 36 years of experience. Since 2017, the unit has on average 119 acute SAH admissions per year. $48 \%$ of these patients receive endovascular treatment, $20 \%$ microsurgery, $3 \%$ both and $29 \%$ have no treatment (including perimesencephalic SAH and moribund patients). In addition, 10.5 elective surgical aneurysm treatments and 45 elective endovascular treatments occur yearly.

Between November 2012 and September 2018, 137 patients presented with a SAH secondary to an ACOM aneurysm. $82.5 \%$ were treated endovascularly and $13.9 \%$ surgically, comparable to $12.5 \%$ in other series (Alleway et al. 2013). There were 187.5 patient-years of follow-up, with a median of 3 years (range $0-73$ months). Rates of vasospasm, cerebral infarction, CSF diversion, rebleed and length of stay were not significantly different in the two cohorts. There 
was no difference in functional outcomes and the majority of patients had a favourable Glasgow Outcome Score of five (42.1\% versus $65.7 \%$ respectively). The 'ACOM syndrome' is a phenomenon of cognitive deficits such as memory impairment and personality changes. One study demonstrated that patients treated with coils have fewer cognitive deficits than those treated surgically (Chan et al. 2002). The results were not significant in our analysis. Recurrence was higher in the coiled cohort with 15 patients requiring further treatment. In a subgroup group analysis of coiled aneurysms, there was no statistical differences in rates of late rebleed or functional outcome in those that recurred and those that did not.

Initial surgical treatment resulted in a $94.7 \%$ complete occlusion and endovascular treatment in $80.5 \%$. Meta-analysis identified a rate of near complete ( $>95 \%)$ occlusion rate immediately after coiling of 88\% (Proust et al. 2003, Finitsis et al. 2010, Fang et al. 2014, Dellaretti et al. 2017). The natural history of aneurysm neck remnants after coil treatments has been largely characterised as benign, however there is a risk of rebleeding (Hayakawa et al. 2000, CARAT Investigators 2006, Henkes et al. 2006, Fang et al. 2014).

Recurrence in coiled aneurysms may occur due to coil compaction from arterial blood flow and true persistent growth of the aneurysm due to the initial disease of the arterial wall (Cognard et al. 1999). Once coil compaction occurs, the arterial blood flow may produce neck remnant growth. Coil migration into intra-aneurysmal thrombus may also be involved (Henkes et al. 2006). Factors identified as significant predictors of recurrence include suboptimal initial angiographic results, treatment after rupture, age, increased aneurysm size and duration of follow-up (Cognard et al. 1999, Raymond et al. 2003, Finitsis et al. 2010). Our study supports these findings. Recurrences following clipping have been well described in the literature (Sakaki et al. 1994, Proust et al. 2003, Ihm et al. 2010, Dellaretti et al. 2017). 
A limited number of studies have examined outcomes and complications specific to ACOM aneurysms. In a 2014 meta-analysis of 1552 endovascularly treated ACOM aneurysms the rate of complete or near complete ( $>95 \%$ ) aneurysm occlusion at six months was $85 \%$ (Fang et al. 2014). Our series looks exclusively at ruptured ACOMs, and does not distinguish between major and minor recurrences and hence our rates are expectedly higher. In our series, recurrences occurred up to 29 months, thus extended follow-up is mandatory (Cognard et al. 1999, Raymond et al. 2003).

Our rate of rebleeding was $5.3 \%$ following coiling (six patients, five rebled early and one late) and $0 \%$ following clipping. A comparison with ISAT is not possible (Molyneux et al. 2002, 2015) due to our small surgical sample, patient demographics, patient selection and our specific focus on ACOM aneurysms. The risk of rebleeding is significantly higher for recurrent aneurysms than for stable aneurysms (Molyneux et al. 2002, Johnston et al. 2008). The decision to manage a recurrent aneurysm conservatively or with further treatment must balance the risks of rebleeding with the risk of re-treatment

Reports of retreatment rates range from 3.5-34\% (Henkes et al. 2006, Sedat et al. 2012). The retreatment rate was $7 \%$ on meta-analysis, which doubled from $5 \%$ to $10 \%$ when comparing studies published before and after 2007 (Fang et al. 2014). This may be due to the development of stents, allowing treatment of some previously untreatable aneurysm recurrences, which would have otherwise been managed with surveillance. The retreatment rate was $10.6 \%$ in our centre. In a series of 2360 coiled aneurysms at different locations, 350 (12.3\%) had retreatments, with a low associated morbidity and mortality rate of 2.2\% (Henkes et al. 2006). Of the 556 ACOM aneurysms, $62(11.2 \%)$ had second treatments and $15(2.7 \%)$ had third. 
Studies consistently shows that retreatment is associated with a low morbidity and mortality (Slob et al. 2004, Sedat et al. 2012).

The decision to retreat was taken by the MDT following radiological progression in the size of the recurrence or neurological symptoms. Criteria for the indication of retreatment does not exist, and decision making is based on individual experience (Raymond et al. 2003). Authors propose to retreat when angiographic control worsens, if the neck is not covered by coils greater than $2 \mathrm{~mm}$, or if there is mass effect and recurrent clinical symptoms (Henkes et al. 2006, Sedat et al. 2012). A randomised controlled trial of recurrent aneurysms comparing retreatment with conservative management if there is clinical equipoise would help to answer these questions.

There are several limitations to our study. It is a retrospective, observational study in a single institution. The individual experience of the treating clinicians and ancillary teams, and local practices could influence outcomes. The sample size was small for the clipped cohort. The follow-up duration was limited (median 33.2 months), and differed between the two treatment arms. Long-term recurrences may occur and may warrant additional treatment. Continuous improvement of interventional devices, in conjunction with increased expertise of our interventional neuroradiologists and neurovascular surgeons from 2012 to 2018 cannot be appreciated in this study. The frequency and intervals for follow-up, though standardised, was not systematic. Despite this, we believe that our study represents an accurate review of immediate and long-term outcomes after treatment of ACOM aneurysms. A prospective study with a longer follow-up on a larger scale is necessary to draw conclusions.

\section{Conclusions}


Patients treated with coiling have an increased risk of residual and recurrent ACOM aneurysms compared to those treated with surgical clipping. However, this does not translate into a difference in morbidity. Rates of vasospasm, cerebral infarction, CSF diversion, rebleed, length of stay, and functional outcome were not significantly different. The impact of recurrence and residual aneurysms remains incompletely understood. Patients should have long-term clinical and angiographic follow-up and treatment decisions should be considered carefully in a multidisciplinary setting.

\section{Acknowledgments}

We acknowledge the neurovascular department at Queen's Hospital Romford, notably the interventional radiologists Dr Raed Alkilani, Dr Sanjiv Chawda and Dr Shahram Derakhshani for their invaluable contribution in managing the study patients.

\section{Funding}

No funding was received for this research

\section{Disclosure of interest}

The authors report no conflicts of interest.

\section{Ethics approval}




\begin{abstract}
All procedures performed in studies involving human participants were in accordance with the ethical standards of the institutional and/or national research committee and with the 1964 Helsinki declaration and its later amendments or comparable ethical standards.
\end{abstract}

For this type of study formal consent is not required

\title{
References
}

Alleway, R., Ellis, D., Freeth, H., Jarman, D., Kelly, K., Nwosu, E., Protopapa, K., Smith, N., and Warsame, A., n.d. Managing the Flow? A report by the National Confidential Enquiry into Patient Outcome and Death (2013).

CARAT Investigators, 2006. Rates of Delayed Rebleeding From Intracranial Aneurysms Are Low After Surgical and Endovascular Treatment. Stroke, 37 (6), 1437-1442.

Chan, A., Ho, S., and Poon, W.S., 2002. Neuropsychological Sequelae of Patients Treated with Microsurgical Clipping or Endovascular Embolization for Anterior Communicating Artery Aneurysm. European Neurology, 47 (1), 37-44.

Cognard, C., Weill, A., Spelle, L., Piotin, M., Castaings, L., Rey, A., and Moret, J., 1999.

Long-term Angiographic Follow-up of 169 Intracranial Berry Aneurysms Occluded with Detachable Coils. Radiology, 212 (2), 348-356.

Dellaretti, M., da Silva Martins, W., Dourado, J., Faglioni, W., Quadros, R., de Souza Moraes, V., and de Souza Filho, C.A., 2017. Angiographic and epidemiological characteristics associated with aneurysm remnants after microsurgical clipping. Surgical 
Neurology International, 8 (1), 198.

Fang, S., Brinjikji, W., Murad, M.H., Kallmes, D.F., Cloft, H.J., and Lanzino, G., 2014.

Endovascular Treatment of Anterior Communicating Artery Aneurysms: A Systematic

Review and Meta-Analysis. American Journal of Neuroradiology, 35 (5), 943-947.

Finitsis, S., Anxionnat, R., Lebedinsky, A., Albuquerque, P.C., Clayton, M.F., Picard, L., and Bracard, S., 2010. Endovascular Treatment of ACom Intracranial Aneurysms. Interventional Neuroradiology, 16 (1), 7-16.

Hayakawa, M., Murayama, Y., Duckwiler, G.R., Gobin, Y.P., Guglielmi, G., and Viñuela, F., 2000. Natural history of the neck remnant of a cerebral aneurysm treated with the Guglielmi detachable coil system. Journal of Neurosurgery, 93 (4), 561-568.

Henkes, H., Fischer, S., Liebig, T., Weber, W., Reinartz, J., Miloslavski, E., and Kühne, D., 2006. Repeated Endovascular Coil Occlusion in 350 of 2759 Intracranial Aneurysms: Safety and Effectiveness Aspects. Neurosurgery, 58 (2), 224-232.

Ihm, E.-H., Hong, C.-K., Shim, Y.-S., Jung, J.-Y., Joo, J.-Y., and Park, S.-W., 2010.

Characteristics and Management of Residual or Slowly Recurred Intracranial Aneurysms. Journal of Korean Neurosurgical Society, 48 (4), 330.

Johnston, S.C., Dowd, C.F., Higashida, R.T., Lawton, M.T., Duckwiler, G.R., Gress, D.R., and CARAT Investigators, 2008. Predictors of Rehemorrhage After Treatment of Ruptured Intracranial Aneurysms. Stroke, 39 (1), 120-125.

Kirkpatrick, P., Lindsay, K., Shaw, M., Gholkar, A., Molyneux, a, and Langham, J., 2006. National Study of Subarachnoid Haemorrhage. The Royal College of Surgeons of England.

Li, H., Pan, R., Wang, H., Rong, X., Yin, Z., Milgrom, D.P., Shi, X., Tang, Y., and Peng, Y., 2013. Clipping Versus Coiling for Ruptured Intracranial Aneurysms. Stroke, 44 (1), 2937. 
Lindgren, A., Vergouwen, M. DI, van der Schaaf, I., Algra, A., Wermer, M., Clarke, M.J., and Rinkel, G.J., 2018. Endovascular coiling versus neurosurgical clipping for people with aneurysmal subarachnoid haemorrhage. Cochrane Database of Systematic Reviews, 8, CD003085.

McDougall, C.G., Spetzler, R.F., Zabramski, J.M., Partovi, S., Hills, N.K., Nakaji, P., and Albuquerque, F.C., 2012. The Barrow Ruptured Aneurysm Trial. Journal of Neurosurgery, $116(1), 135-144$.

Mitchell, P., Kerr, R., Mendelow, A.D., and Molyneux, A., 2008. Could late rebleeding overturn the superiority of cranial aneurysm coil embolization over clip ligation seen in the International Subarachnoid Aneurysm Trial? Journal of Neurosurgery, 108 (3), 437442.

Molyneux, A., Kerr, R., Stratton, I., Sandercock, P., Clarke, M., Shrimpton, J., Holman, R., and International Subarachnoid Aneurysm Trial (ISAT) Collaborative Group, 2002. International Subarachnoid Aneurysm Trial (ISAT) of neurosurgical clipping versus endovascular coiling in 2143 patients with ruptured intracranial aneurysms: a randomised trial. Lancet (London, England), 360 (9342), 1267-74.

Molyneux, A.J., Birks, J., Clarke, A., Sneade, M., and Kerr, R.S.C., 2015. The durability of endovascular coiling versus neurosurgical clipping of ruptured cerebral aneurysms: 18 year follow-up of the UK cohort of the International Subarachnoid Aneurysm Trial (ISAT). Lancet (London, England), 385 (9969), 691-7.

Molyneux, A.J., Kerr, R.S., Birks, J., Ramzi, N., Yarnold, J., Sneade, M., Rischmiller, J., and ISAT Collaborators, 2009. Risk of recurrent subarachnoid haemorrhage, death, or dependence and standardised mortality ratios after clipping or coiling of an intracranial aneurysm in the International Subarachnoid Aneurysm Trial (ISAT): long-term followup. The Lancet Neurology, 8 (5), 427-433. 
Proust, F., Debono, B., Hannequin, D., Gerardin, E., Clavier, E., Langlois, O., and Fréger, P., 2003. Treatment of anterior communicating artery aneurysms: complementary aspects of microsurgical and endovascular procedures. Journal of Neurosurgery, 99 (1), 3-14.

Raymond, J., Guilbert, F., Weill, A., Georganos, S.A., Juravsky, L., Lambert, A., Lamoureux, J., Chagnon, M., and Roy, D., 2003. Long-Term Angiographic Recurrences After Selective Endovascular Treatment of Aneurysms With Detachable Coils. Stroke, 34 (6), 1398-1403.

Sakaki, T., Takeshima, T., Tominaga, M., Hashimoto, H., and Kawaguchi, S., 1994. Recurrence of ICA-PCoA aneurysms after neck clipping. Journal of Neurosurgery, 80 (1), 58-63.

Sedat, J., Chau, Y., Moubarak, K., Vargas, J., and Lonjon, M., 2012. Endovascular Treatment of Recurrent Coiled Aneurysms: Assessment of Complications and Rebleeding during a Decade in a Single Center. Interventional Neuroradiology, 18 (1), 14-19.

Slob, M.J., Sluzewski, M., van Rooij, W.J., Roks, G., and Rinkel, G.J.E., 2004. Additional coiling of previously coiled cerebral aneurysms: clinical and angiographic results. AJNR. American journal of neuroradiology, 25 (8), 1373-6.

Spetzler, R.F., McDougall, C.G., Zabramski, J.M., Albuquerque, F.C., Hills, N.K., Russin, J.J., Partovi, S., Nakaji, P., and Wallace, R.C., 2015. The Barrow Ruptured Aneurysm Trial: 6-year results. Journal of neurosurgery, 123 (3), 609-17. 


\section{$\underline{\text { Tables }}$}

\begin{tabular}{|c|c|c|c|}
\hline Variable name & Clipping $(n=19)$ & Coiling $(n=113)$ & p value \\
\hline Age (years) & $57.9+/-13.6$ & $57.6+/-15.4$ & 0.9174 \\
\hline Female & $12(63.2)$ & $67(59.3)$ & 0.7492 \\
\hline \multicolumn{4}{|l|}{ Comorbidities } \\
\hline Hypertensive & $7(50)$ & $29(49.1)$ & 0.9520 \\
\hline Ischaemic heart disease & $0(0)$ & $3(7.5)$ & 0.3140 \\
\hline Smoker & $4(28.6)$ & $37(57.8)$ & 0.0489 \\
\hline Known ACOM pre & $2(10.5)$ & $0(0)$ & 0.0005 \\
\hline \multicolumn{4}{|l|}{$\mathrm{SAH}$} \\
\hline \multicolumn{4}{|l|}{ WFNS grade } \\
\hline $\mathrm{I}$ & $7(41.2)$ & $62(60.8)$ & 0.1312 \\
\hline II & $2(11.8)$ & $23(22.5)$ & 0.3178 \\
\hline III & $0(0)$ & $5(4.9)$ & 0.3531 \\
\hline IV & $6(35.3)$ & $6(5.9)$ & 0.0002 \\
\hline $\mathrm{V}$ & $2(11.8)$ & $6(5.9)$ & 0.3711 \\
\hline 'Good grade' I+II & $9(52.9)$ & $85(83.3)$ & 0.0046 \\
\hline 'Poor grade' III-V & $8(47.1)$ & $17(16.7)$ & 0.0046 \\
\hline \multicolumn{4}{|l|}{ Fisher grade } \\
\hline 1 & $0(0)$ & $6(7.0)$ & 0.2776 \\
\hline 2 & $1(6.3)$ & $13(15.1)$ & 0.3499 \\
\hline 3 & $4(25)$ & $14(16.3)$ & 0.4044 \\
\hline 4 & $11(68.8)$ & $53(61.6)$ & 0.5863 \\
\hline \multicolumn{4}{|l|}{ Best motor score } \\
\hline 1 & $4(23.5)$ & $4(4.4)$ & 0.0060 \\
\hline 2 & $0(0)$ & $0(0)$ & 0.9999 \\
\hline 3 & $0(0)$ & $1(1.1)$ & 0.6654 \\
\hline 4 & $2(11.8)$ & $3(3.3)$ & 0.1279 \\
\hline 5 & $2(11.8)$ & $3(3.3)$ & 0.1279 \\
\hline 6 & $9(52.9)$ & 80 (87.9) & 0.0005 \\
\hline
\end{tabular}

Table 1; Baseline patient characteristics. Data are given as mean $+/-$ standard deviation, median and range or frequency and percent. P values from unpaired two tailed t-test and chisquared test as appropriate. WFNS = World Federation of Neurological Surgeons. P values 
in bold highlight significant differences. Of note, some patients had data missing from their clinical records.

\begin{tabular}{|llll|}
\hline Variable name & Clipping $(\mathbf{n}=\mathbf{1 9})$ & Coiling $(\mathbf{n}=\mathbf{1 1 3})$ & p value \\
\hline & & & \\
Neck $(\mathrm{mm})$ & $1.9(0.7-3.2)$ & $1.7(0.7-3.8)$ & 0.5049 \\
Dome $(\mathrm{mm})$ & $3.9(1.6-13.0)$ & $4.1(1.5-11.5)$ & 0.8632 \\
Dome: neck ratio & $2.3(0.9-6.8)$ & $2.6(1.2-6.3)$ & 0.8751 \\
Dome: neck ratio $>2$ & $15(78.9)$ & $90(81.1)$ & 0.8280 \\
Maximum diameter $(\mathrm{mm})$ & $3(1.5-10.4)$ & $3.4(0.8-9.5)$ & 0.8263 \\
Size $(\mathrm{mm})$ & $4(1-8)$ & $4(2-16)$ & 0.2356 \\
Parent vessel diameter $(\mathrm{mm})$ & $2(0.8-2.8)$ & $1.8(0.7-2.8)$ & 0.3001 \\
Flow angle (degree) & $136(76-172)$ & $140(65-170)$ & 0.3115 \\
Size ratio & $2.3(0.8-3.8)$ & $2.4(0.9-7.6)$ & 0.1547 \\
Number of lobes & $1(1-3)$ & $1(1-2)$ & 0.1851 \\
Incorporating ACOM artery & $9(47.4)$ & $26(23.4)$ & $\mathbf{0 . 0 3 0 3}$ \\
Fundus projection: posterior & $1(8.3)$ & $1(1.8)$ & 0.2330 \\
Fundus projection: superior & $5(62.5)$ & $24(34.3)$ & 0.1201 \\
& & & \\
\hline
\end{tabular}

Table 2; Baseline aneurysm characteristics. Data are given as median and range or frequency and percent. Flow angle $=$ angle between maximum height of aneurysm and parent vessel. Size ratio $=$ ratio of size to parent vessel diameter. $P$ values from unpaired two tailed $t$-test and chisquared test as appropriate. P values in bold highlight significant differences.

\begin{tabular}{|llll|}
\hline Variable & Clipping $(\mathbf{n}=\mathbf{1 9})$ & Coiling $(\mathbf{n}=\mathbf{1 1 3})$ & p value \\
\hline Time to treat (days) & $1(0-6)$ & $1(0-15)$ & 0.3537 \\
Residual aneurysm & $1(5.3)$ & $22(19.5)$ & 0.1328 \\
& & & \\
Peri-procedural complication & $0(0)$ & $3(2.7)$ & 0.4704 \\
Post treatment seizure & $1(5.6)$ & $3(3.9)$ & 0.7485 \\
& & & \\
Vasospasm & $9(47.4)$ & $29(25.7)$ & 0.0542 \\
Time to vasospasm (days) & $5(0-9)$ & $4.5(1-14)$ & 0.7649 \\
Angioplasty & $7(77.8)$ & $21(72.4)$ & 0.7512 \\
Infarct & $8(42.1)$ & $27(23.9)$ & 0.0976 \\
& & & \\
CSF diversion & $9(47.4)$ & $30(26.5)$ & 0.0656 \\
Shunt & $2(22.2)$ & $6(20.0)$ & 0.8875
\end{tabular}




$\begin{array}{llll}\text { Time to shunt (days) } & 22.5(22-23) & 117.5(23-289) & 0.2610 \\ \text { Rebleed } & 0(0) & 6(5.3) & 0.3062 \\ \text { Early (<30 days) } & & 5(4.4) & \\ \text { Late ( }>30 \text { days) } & & 1(0.9) & \\ & & & \\ \text { Glasgow outcome score (GOS) } & & 67(65.7) & 0.0527 \\ 5=\text { Low disability / Good recovery } & 8(42.1) & 13(9.8) & 0.4400 \\ 4=\text { Moderate disability } & 3(15.8) & 8(7.8) & 0.2666 \\ 3=\text { Severe disability } & 3(15.8) & 0(0) & \mathbf{0 . 0 2 0 1} \\ 2=\text { Persistent vegetative state } & 1(5.3) & 14(13.7) & 0.4070 \\ 1=\text { Death } & 4(21.1) & & \\ & & 80(78.4) & 0.0586 \\ \text { Favourable GOS (4 }+5) & 11(57.9) & 7(6.2) & 0.8796 \\ \text { Cognitive / memory problems } & 1(5.3) & 14(2-77) & 0.0676 \\ & & 66(63.5) & 0.0810 \\ \text { Length of stay (days) } & 24(1-41) & 11.5(3-57) & 0.8085 \\ \text { Discharged home } & 8(42.1) & & \\ \text { Time to death (days) } & 28(1-36) & 22(19.5) & 0.1317 \\ & & 23(36.5) & \mathbf{0 . 0 4 3 3} \\ \text { Residual } & 1(5.3) & 6(0-29) & 0.1791 \\ \text { Recurrence at 1 year } & 1(7.7) & 15(32.6) & 0.3353 \\ \text { Time to recurrence (months) } & 4(4) & 16(1-54) & \\ \text { Retreatment } & 0(0) & & \\ \text { Time to retreatment (months) } & & & \end{array}$

Table 3; Complications and outcomes. Data are given as median and range or frequency and percent. $P$ values from unpaired two tailed $t$-test and chi-squared test as appropriate. $P$ values in bold highlight significant differences. Of note, some patients had data missing from their clinical records.

\begin{tabular}{|llll|}
\hline Variable & No recurrence $(\mathbf{n}=\mathbf{6 9})$ & Recurrence $(\mathbf{n}=\mathbf{2 8})$ & p value \\
\hline Age (years) & $57.5+/-15.6$ & $50.9+/-12.0$ & $\mathbf{0 . 0 4 6 6}$ \\
Female & $40(58.0)$ & $15(53.6)$ & 0.6934 \\
Smoker & $24(34.8)$ & $11(39.3)$ & 0.6774 \\
Aneurysm size $(\mathrm{mm})$ & $4(2-11)$ & $5(2-16)$ & $\mathbf{0 . 0 0 3 9}$ \\
& & & \\
Late Rebleed & $0(0)$ & $1(3.6)$ & 0.1150 \\
Favourable GOS $(4+5)$ & $53(91.4)^{*}$ & $27(96.4)$ & 0.3891 \\
\hline
\end{tabular}


Table 4; Subgroup analysis on aneurysms that recurred after coiling versus those that did not. This analysis necessarily excluded deceased patients. Data are given as mean +/- standard deviation, median and range or frequency and percent as appropriate. P values from unpaired two tailed t-test and chi-squared test. GOS = Glasgow outcome score. $P$ values in bold highlight significant differences. * Data only available for 58 patients.

\begin{tabular}{|l|l|l|l|l|l|l|l|}
\hline Patient & Sex & $\begin{array}{l}\text { WFNS } \\
\text { score }\end{array}$ & $\begin{array}{l}\text { Age at } \\
\text { SAH }\end{array}$ & $\begin{array}{l}\text { Dome to } \\
\text { neck ratio }\end{array}$ & Occlusion & GOS & $\begin{array}{l}\text { Day of } \\
\text { rebleed }\end{array}$ \\
\hline 1 & F & 1 & 85 & 3.3 & Complete & 4 & 2 \\
\hline 2 & F & 1 & 73 & 3.4 & Complete & 1 & 4 \\
\hline 3 & F & 5 & 50 & 2.3 & Complete & 1 & 6 \\
\hline 4 & F & 1 & 64 & 2.3 & Residual & 5 & 8 \\
\hline 5 & F & 4 & 76 & 2.9 & Residual & 5 & 13 \\
\hline 6 & M & 1 & 43 & 2.7 & Recurrence & 5 & $\mathbf{4 3 3}$ \\
\hline
\end{tabular}

Table 5; Characteristics and time to rebleed for the patients who rebled. $F=$ female, $M=$ male, WFNS $=$ World federation of neurological surgeons, SAH =Subarachnoid haemorrhage, GOS = Glasgow Outcome score, $M D T=$ multidisciplinary team . 
We thank the editor and reviewers for their further comments. We have addressed these in a point by point fashion below. We hope this has improved the manuscript and that you now find it suitable for publication.

\section{Reviewer: 1}

Comments to the Author

Thank you for making the changes and highlighting them. I can recommend publication.

Thank you.

\section{Reviewer: 2}

Comments to the Author

Thanks for sending the revised manuscript. I am grateful for the authors on further revision suggested by previous reviewers and resubmission of manuscript.

I have the following comments and suggestions.

Title : Page 1, Line 3 (then on Line/Page - 3/1)

Title is too generic - it needs to me more specific as suggested in the introduction of the abstract i.e. comparison between outcomes of endovascular and surgical treatments of ruptured ACOM aneurysms

We have changed the title as recommended.

15/3: I assume the research question posed is "what is the natural history and optimal treatment for recurrent ACOM aneurysm?' 22/4: The conclusion in the abstract - does not answer this question (i.e. remains unknown) and rightly points out that all treatment recommendations should be considered carefully (my suggestions for wording would be "Hence, treatment decisions should be taken by patients after they have been given carefully considered recommendations from the multi-disciplinary team'.

We have made this change as recommended.

$13 / 5$ - up to $40 \%$ figure seems to be referenced to Fang et al which in turn is referenced to an NEJM article which does mention $30 \%$ figure for acom aneurysm in a picture.

Thank you for pointing this out. We have made the correction.

3/7 - was GOS outcome based on structured interview from patients or relatives?

All patients were clinically evaluated before hospital discharge and informally in outpatient clinic follow-up. Cognitive and memory deficits were identified by the therapy teams at discharge and formally assessed on neuropsychology follow-up. This was assessed 
informally, mostly lead by our therapy teams. We have added this into the text for clarification.

$6 / 7$ - one of the key results in the manuscript is that recurrence was observed in $41.4 \%$ of aneurysms in coiled group. This required more exploration in methodology - i.e the line "residual or recurrence was diagnosed from imaging by the MDT to reduce intra-observer variability" is not sufficient. It is retrospective review of clinical records? There are well defined criteria for recurrence of aneurysms post treatment and immediate post treatment appearances such as Raymond Roy scale. was the follow up imaging blindly assessed by investigators (or neuroradiologists) for recurrences/residual aneurysms or was the information extracted from previously issued reports. There are more comments about this matter below

We agree this point would benefit from more discussion, we have addressed this further below. We have reviewed our original data and changed our analysis to reflect only recurrent aneurysms. Thank you for highlighting this to us - we hope this improves the manuscript. We have changed the text below.

Data on residual or recurrent aneurysms was extracted from imaging reports by consultant neuroradiologists. If the information was not readily available, a further imaging review was done by the MDT to reduce intra-observer variability.

$22 / 8$ - why is this presumed? this information is readily available in line starting at $33 / 8$ where I can count 2 haematomas out of 19 - even if one takes these 2 were in poor grade still it makes it only 2 out of 8 clipping indications. Best would be to provide alternative explanation for more proportion of poor grade patients in clipping group. Another indication for clipping is stated to be wide neck or very small aneurysms but in the table 2 - all the variable of aneurysm characteristics are well matched and in fact more proportion of wide neck aneurysms were coiled. The only statistically significant number is for incorporation of acom artery where also quarter of coiled aneurysm had this feature.

You are correct. This sentence has been removed - many thanks for pointing this out. In the results section we now only state the difference rather than speculate a reason.

In the table 1 (3/23) - WFNS data is only given for 17 of the 19 clipped patients and 102/113 coiling patients. These numbers should be the same - explanation for missing WFNS grade in missing 13 patients.

Unfortunately, we were unable to identify all the data in the medical records. Some records were paper and documentation was imperfect. We have clarified this in the Table legend.

38/8 - The two sentences are not required (Currently.. to develop) - does not add to the study as all patients were treated within an agreed time interval. Readers can make an assumption that treating within 48 hours by clipping if coiling is not available is an acceptable indication 
for clipping. Weekend coiling (and clipping) service is available in very few centres in the UK (I assume most of the readers are from UK and they would be well aware of this). Hope for a weekend coiling service on the basis of hope for a future thrombectomy service is not required in the manuscript. This also somehow gives a notion that if weekend coiling was available less patients would have undergone clipping.

Thank you for pointing this out. We agree and both sentences have been removed.

54/8 - was stent placement discussed before coiling was considered the best preventive strategy for re-bleed? this contradicts with a line (3/13) below that stent placement is not advocated in the centre. Why weren't these three patients clipped?

Whilst we don't generally advocate stents acutely, we do consider stents for recurrences. The decision to do so in three cases was taken following MDT discussion between the Interventionalists and Neurosurgeons. Two of these patients died on day 4 and 15 and the other made a good recovery. We have changed the text to reflect this.

10-14/9 - please clarify and correct - immediate assessment of aneurysm for coiling should be the immediate periprocedure last angiogram (i.e. DSA) or immediate baseline MRA - CTA cannot make this assessment, for clipping only DSA (and upto an extent CTA recently, but not MRA). Did all patients in clipping group undergo a post-operative DSA?

Yes. This was assessed on immediate peri-procedural DSA for the coiled group, and postoperative DSA (53.8\%) / CTA (30.8\%) / MRA (15.4\%) for the clipped group. This has been corrected in the text.

47/9 - did optimum medical management include induced hypertension?

Yes. If deemed appropriate by the neurosurgical and neurocritical care teams. This has been added to the text.

Chemical or mechanical angioplasty (line above) - is not supported in table 3 which only mentions chemical angioplasty - please correct.

This has been corrected - many thanks.

$52 / 9$ - what is the correlation with groups which received angioplasty? i.e. did angioplasty help?

Restitution of the calibre of the vessel was obtained in all angioplasty cases, as determined by intra-operative angiography. This has been clarified in the text. 
There were no immediate complications after surgery but $42 \%$ patient had radiologically diagnosed infarcts? this needs some explanation.

We removed the sentence regarding no immediate surgical complications and ammended the text for clarity. 9 surgical patients and 29 coiled patients had symptomatic vasospasm, and 8 out of 27 had infarcts seen on CT or MRI, secondary to this.

In the coiled cohort, three patients (2.7\%) had peri-procedural complications, including one aneurysm rupture (0.9\%), and two thromboembolic events (1.8\%) resulting in transient neurological deficits (Table 3).........Eight surgical (42.1\%) and 27 endovascular (23.9\%) patients had radiological evidence of infarcts.

15/10 - Outcomes -

Text vs Table 2

If you divide this figure in two groups in the text (from table line 11/25) - Favourable outcome (GOS 5/4) - surgical group $11 / 19$ - and say $58 \%$ had favourable outcome and $78 \%$ in coiling group (80/102). Please give a reason for no data for 11 patients in coiling group. The line "majority of patients had favourable outcome is best for readers to decide"

Thank you for pointing this out. We agree and have corrected this in the text. Unfortunately, some data was missing from the patient's clinical record and this has been noted in the legend.

Of note, some patients had data missing from their clinical records.

54/10 Follow up

You have indicated immediate follow up for clipped patients above - there is some confusion - did clip patients have another follow up? Please remove MRA and CTA if they were not used for follow up in clipped group).

Thank you for pointing this out, we have added in post-operative imaging data.

For coiling group please indicate modality - MRA or DSA but unlikely to be CTA.

Usually MRA - This has been corrected in the text

Clipped patients with no residuals - is it based on immediate post op DSA or one done later.

Long term follow up for clipped patients is not available/not performed. So later on comparison of delayed recurrences will not be possible. Essentially the study becomes single 
arm for coiling patients with regards to aneurysm follow up. This also will mean revising the introduction line15/3.

We agree with this and have revised the text to remove the direct comparison

This study looks at the rates of complications and recurrences of ruptured ACOM aneurysms treated endovascularly and surgically.

$17 / 11$ - the sentence "There was a statistically significant increase in the risk of ACOM recurrence in the coiled group when compared to the clipped group $(p=0.014)$ " is based on clipped group mean follow up from 1 to 4 months vs residuals left at the time of treatment plus true recurrences on follow up ranging from 0-29 months in the coiling group. This therefore is not like to like comparison and inaccurate. It is well known that coiled aneurysms have a recurrence rate, retreatment rate and therefore these are followed up.

19 genuine recurrences have been clubbed with 27 post treatment residuals (were these grade 2 ? - i.e .grade 1 and grade 2 is considered satisfactory occlusion post coiling or grade 3). Recurrence by definition cannot happen immediately post treatment.

Thank you for giving us the opportunity to correct this important point. We have gone back to our original data and changed how we have analysed our results as a result.

1) We have included recurrences only and not residuals.

2) For residuals we have classified them as per Raymond Roy.

3) We have drawn a like for like comparison and only compared the results at 1 year.

4) We have cut down the text considerably to make this clearer and more concise

5) We have adjusted our KM curve to reflect this.

6) The abstract / table 3 has been changed to reflect this

7) We have changed our subgroup analysis to reflect this (Table 4)

Abstract: There was a statistically significant increase in the risk of ACOM recurrence in the coiled group when compared to the clipped group at one year $(p=0.0433) .15$ patients required further treatment at a median time of 16 months

Treatment: Complete obliteration (no filling of contrast medium in the dome, body or neck) was achieved in $94.7 \%$ of the clipped patients (one patient had a residual) and $80.5 \%$ in the coiled group. 22 of the coiled cohort had a residual, 18 (81.8\%) were Raymond Roy classification II and 4 (18.2\%) Raymond Roy classification IIIb, assessed on immediate periprocedural DSA. No patients had a planned two-stage approach. For the clipped cohort, imaging modality depended on anatomical and patient specific considerations and included DSA $(53.8 \%)$, CTA $(30.8 \%)$ or MRA (15.4\%).

Follow-up: There was a statistically significant increase in the risk of ACOM recurrence in the coiled group when compared to the clipped group at one year $(p=0.0433)$. Recurrences ranged from immediately post treatment up to 29 months (Figure 3). 15 aneurysms that 
recurred after coiling were re-treated, 14 endovascularly (including coils, flow diverter or web as appropriate) and one surgically, at a median time of 16 months. 10.6\% of the initial cohort of coiled patients had a second procedure. No patients have yet had a third procedure although follow-up continues. Recurrence over time for the coiled cohort can be seen in Figure 4.

Subgroup analysis of coiled aneurysms that had a recurrence versus those that did not showed that younger age $(p=0.0466)$ and larger aneurysm size $(p=0.0039)$ were associated with recurrence.

\begin{tabular}{|llll|}
\hline Variable & No recurrence $(\mathbf{n}=\mathbf{6 9})$ & Recurrence $(\mathbf{n}=\mathbf{2 8})$ & p value \\
\hline Age (years) & $57.5+/-15.6$ & $50.9+/-12.0$ & $\mathbf{0 . 0 4 6 6}$ \\
Female & $40(58.0)$ & $15(53.6)$ & 0.6934 \\
Smoker & $24(34.8)$ & $11(39.3)$ & 0.6774 \\
Aneurysm size $(\mathrm{mm})$ & $4(2-11)$ & $5(2-16)$ & $\mathbf{0 . 0 0 3 9}$ \\
& & & \\
Late Rebleed & $0(0)$ & $1(3.6)$ & 0.1150 \\
Favourable GOS $(4+5)$ & $53(91.4)^{*}$ & $27(96.4)$ & 0.3891 \\
\hline
\end{tabular}

Similarly it is not unusual to finish coiling providing dome/bleeding point protection and address full treatment later as a planned stage 2 procedure. Some of these may have been included in re-treated group. If some aneurysms were treated with two stage approach - this should be clarified.

We have re-reviewed the data and no aneurysms were treated with a two-stage approach.

Early rebleed (line $31 / 12$ ) $-5.3 \%$ is on the higher side of reports (2\% ISAT) and numbers are big enough in this series. Some insight into this will be helpful.

Our early rebleed rate was $4.4 \%$ - we agree, and we are unsure why this was higher than other reports. The demographics were included in table 5 for transparency. We have added in sex, age and dome to neck ratio to add further insight. We offer an explanation in the discussion.

Discussion

$3 / 13$ - please see stent comment above

This has been changed as noted above

$10 / 13$ - I understand this paragraph has been added by suggestions of previous reviewer. I think this is too long.

Thank you, we have reduced the length of this paragraph. 
$33 / 13$ - please qualify that mean ?imaging follow up for coiled group was 33.2 months - not for clipped patients.

This referred to the whole cohort, this has been clarified in the text ie There were $\mathbf{1 8 7 . 5}$ patient-years of follow-up, with a median of 3 years (range 0-73 months).

54/13 - add late re-bleed (not re-bleed only) to differentiate from early re-bleed a term used above - I think in the study there was one late rebleed in a patient who did not attend follow up imaing?

This has been corrected in the text - thank you

$4 / 14$ It is a moot point but 'goal of treatment is to prevent a re-bleed'.

This has been corrected in the text - thank you

22 and 24/14 are hypothesis/assumptions and that should be clarified.

We have clarified this in the text - thank you

$52 / 14$ - this has been addressed here in discussion but I would expect the study to distinguish it particularly when conclusions have been heavily drawn from this data set. An explanation would be required in discussion.

6/15 - paragraph about re-bleeding and table 4

Both these are confusing -

There were 5 early re-bleeds, ( 3 had full occlusion, 2 had residual necks left post

treatment). 2 of these patients died.

1 was a late re-bleed at day 433 in a patient who missed follow ups

The same info in table 4 is as follows

2 out of 3 who had full occlusion post coiling died - that left 1 patient which is included in "no recurrence group" and 2 early re-bleeds with residual necks on immediate post coiling and 1 later re-bleed - the patient who missed follow up, has been added in the recurrence group.

This methodology of showing results have led $2.3 \%$ re-bleed with no recurrence and $6.5 \%$ with recurrence.

I think all these figures in discussion make interpretation very difficult.

Many thanks for these points, we accept them all and as a result we have changed our statistical analysis and manuscript to reflect your comments. We hope you find it clearer and much improved now.

We have only included true recurrences (not residuals) - as above. Table 4 now only includes late rebleeds 
The only hard conclusion from this study is simply - the early re-bleed rate was $5 / 113$ in coiling group and 1 delayed re-bleed - could have been prevented, had the patient attended the follow up. The methodology of study does not allow other conclusions. Main flaw being the definition of what is constituting a recurrence.

Many thanks. We have changed our definition to include only recurrence (not residuals) to clarify our conclusions and their limitations.

Comparison with ISAT - is not possible primarily due to low numbers in surgical arm, patient selection (clinical practice vs randomised study).

We agree and have amended the text to reflect this point. Specifically:

A comparison with ISAT is not possible (Molyneux et al. 2002, 2015) due to our small surgical sample, patient demographics, patient selection and our specific focus on ACOM aneurysms.

Re-treatments - a statement somewhere why retreatments increased after 2007 - it may be due to development of stents - a lot of aneurysm recurrences which prior to this date would not be treatable and therefore would only be followed up - can since then be treated. Most centres had a rapid upward curve of re-treatment of long waiting untreatable recurrent aneurysms due to introduction of numerous adjunct devices, which is now levelling off.

Thank you for this comment. We have incorporated it as below. This may be due to the development of stents, allowing treatment of some previously untreatable aneurysm recurrences, which would have otherwise been managed with surveillance

$34 / 16$ - I am not sure info about having a bypass facility affects this study - it will be highly unsual for a bypass to be used for an ACOM aneurysm and therefore even if this facility existed - the impact on study results will be none to minimal. The largest aneurysm in the series was $13 \mathrm{~mm}$. I understand this line has been added from advice from previous reviewer.

We agree and this has been deleted

Pan London rota for surgeons and 24/7 endovascular treatment are largely irrelevant to this study as authors managed to follow current NCEPOD recommendations ( 1 am not sure if treating aneurysms within 48 hours is mandatory though - if it was most centres in UK would be covering a weekend coiling/clipping service).

We agree and this has been deleted

Comment on the last line (38/16) and my overall comments -

the paper and meta-analysis by Fang gives review of endovascular treatment of acom 
aneurysms with high number of patients. High early re-bleed is not reflected in literature to this extent and this study has $>100$ patients treated in endovascular arm. Clipping 8/19 patients in grade IV and $V$ within 48 hours would be unusual for most centres especially when only 2 patients needed clot evacuation. One important concern affecting the conclusion is including all immediate post coil residuals appearances as recurrences, not differentiating minor and major recurrences, when in reality some of these could have been a grade 2 appearance and satisfactory. I think therefore this study is less than accurate review of ruptured acom aneurysms.

Thank you for your expert critique and feedback. We have changed our analysis on your direction and are grateful for your help in improving the manuscript. We now only look at true recurrences (and not residuals as per previous).

\begin{abstract}
Authorship - I note that three endovascular operators have been acknowledged in this paper but have not been included in authorship. If the contents of the data used in the paper was drawn from their work in coiling, angioplasty, post coil/clip DSAs, and reports of non-invasive imaging, then that data is their intellectual input and therefore lead author should consider their role in authorship.
\end{abstract}

Many thanks. You are of course correct that the input of our highly valued endovascular colleagues input into the care of these patients was absolutely essential. However, they did not contribute to any aspect of the study design, research conduct, or manuscript preparation. Therefore, we felt an acknowledgement was more appropriate than full authorship as per ICMJE guidelines. They are aware of our use of the data and we have outlined the invaluable importance of their contribution in the acknowledgement.

Associate Editor's Comments to Author:

Associate Editor

Comments to the Author:

Please see reviewer comments. Reviewer 2 has added a lot of detailed comment and suggestions. Please consider incorporating some of this.

Many thanks, we have substantially revised the manuscript to address the reviewer comments. We hope you find it much improved, and of high quality and suitable interest for the readership of BJNS. 


\section{Managing Ruptured Anterior Communicating Artery Aneurysms -}

\section{Responses to Reviewer Comments}

Many thanks for your comments. We have addressed each one in turn below and made the required changes. Thank you for the opportunity to improve the manuscript. We hope it is now appropriate for publication in the BJNS.

\section{Reviewer: 1}

Comments to the Author

I appreciate the authors effort in crafting this manuscript. Although this manuscript captures the experience of a single centre, and for that it has its own merit and descriptive power, I am concerned that the low sample size in the microsurgical group 19 patients in 6 years may account for some of this paper's findings (eg remnant after clipping).

IT would be informative to know the overall clipping cases and endovascular cases per year (accumulated experience ruptured and unruptured, to determine experience and skill from the physicians)

Agreed - we have added this data into the discussion as documented below. We have also recognised the small sample size for the clipped cohort in our limitations

Our unit is made up of two consultant vascular neurosurgeons (with 17 years combined experience) and three interventional neuroradiologists (with 36 years combined experience). Since 2017, the unit has on average 119 acute admissions per year secondary to a SAH. 48\% of these patients receive endovascular treatment, $20 \%$ microsurgery, $3 \%$ receive both and $29 \%$ have no treatment following a DSA (this includes perimesencephalic SAH and patients not fit for intervention). In addition, 10.5 elective surgical aneurysm treatments and 45 elective endovascular treatments occur each year.

Was ICG intraoperative video angiography used when clipping?

Yes - We have added this in.

Was bypass considered for those large aneurysms that were assigned typically in the endovascular group? If not, please explain why in the discussion section.

At the time of data collection bypass was not performed in our unit. If it were felt to be required then the option of referral to another centre with this capacity would have been possible. Bypass is now offered in our unit - we have added this into the limitations paragraph of the discussion.

For this article to be of impact to the literature, the authors need to provide a table with the full features of the sample (e.g neck to dome ratio $>$ or $<2$, aneurysm size, anatomical geometry of the aneurysm, use or not of stent assisted coiling, mean time to treat -door to proedure-, GCS at presentation, rebleed day... for EACH group).

Many thanks - we have gone back to our sample and collected additional data including size of aneurysm neck, dome, neck to dome ratio, maximum diameter, size, parent vessel diameter, flow 
angle, size ratio, number of lobes, if the aneurysm incorporated the ACOM arteries, the direction of the fundus and whether a stent or flow diverter was used. This data has been shown in the new table 2 and discussed in the results section as seen below. Best motor score and WFNS grade is still included in table 1. Time to treat is in table 3. Rebleed day is in table 5. Thank you for highlighting this.

The aneurysm characteristics were well matched in both cohorts (Table 2), including size of neck, dome, dome to neck ratio, maximum diameter, size, parent vessel diameter, flow angle, size ratio, number of lobes and fundus projection. The aneurysm incorporated the ACOM artery more frequently in the clipped cohort ( $47 \%$ versus $23 \%, p=0.0303$ ).

Of the 113 patients treated endovascularly, three patients required a stent on initial treatment, and none required a flow diverter.

The way Figure 3 is presented is not informative and misleading, looks like $90 \%$ of clipped aneurysms recur? please present this data with \% of recurrence in the $\mathrm{Y}$ axis and time in $\mathrm{X}$ (in the text you mention you did not follow clipped more than 1 year but the table goes on for 5 years!!!!)

Thank you - we have made both changes as suggested above to figure 3 (thank you for highlighting our error)

There are grammatical and style errors, see line 40 "they are can be..."

This has been corrected. We have reviewed the remaining text grammatical and style errors.

Overall this article has value but these points must be addressed to be of merit.

Thank you. 


\section{Reviewer: 2}

Comments to the Author

The authors are to be commended for their detailed work and analysis. This is a realistic account of the management of ACOM aneurysm in a UK neurosurgical unit and is of value to the readership. However, the manuscript does suffer from a few flaws, which need to be clarified:

1. Two cases in the surgically treated group were in patients with known ACOM aneurysms under surveillance with a plan to treat endovascularly if required, versus none in the endovascular group $(p=0.0005)$ - I am unclear about this; why if the prior decision was for endovascular coiling - were these treated by clipping?? Perhaps a note to clarify would be helpful.

Thank you for the valuable feedback. We have clarified and expounded on this in the text. The initial decision was to 'treat as required' and the final decision to clip was made in neurovascular MDT. We hope this is now clear in the text.

2. As is the case in most UK centres, there is no regular weekend endovascular service, this means, in order to treat within 48 hours, some patients are offered clipping. - Did the authors consider networking at any point? Was there a system of this nature ever in place?

Yes, this important point was considered. As the reviewer is aware, this is continually changing situation - and indeed has changed during the study with the introduction of a pan-London on call rota for neurovascular consultant cover. Likewise, there is some momentum to introduce this for the endovascular service too. These points are now briefly addressed in the text.

3. Complete obliteration - on DSA postop - Is it the normal protocol for all clipped patients to have a DSA post op in the unit?

All clipped patients have a formal DSA or CTA. This is now described in the text (see below for extract).

All clipped patients had a post-operative angiogram- either a CTA or DSA depending on anatomical and patient specific considerations.

4. No peri-treatment complications were recorded in the clipping cohort However, 8 surgical (42.1\%) and 27 endovascular (23.9\%) patients had radiologically diagnosed infarcts. - These two statements don't quite add up; what is the authors' definition of 'peritreatment' complications? Infarcts and spasm would technically count as peri treatment problems.

Apologies for the contradictory statements. This has been reworded for clarity. The intention was of the word 'peri-treatment' was to describe complications occurring during or immediately after the treatment. Thank you for pointing out that this term is not widely recognised. This has now been corrected in the text.

5. Mortality rate was $21.1 \%$ in the clipping and $13.7 \%$ in coiling on median day...- This needs to be stratified by initial grade of presentation, otherwise these numbers don't make much sense.

\section{This has been included.}

There was no difference on stratifying mortality by initial grade of presentation (100\% of the clipped cohort and $66.7 \%$ of the coiled cohort who died presented with poor grade WFNS III-V, $p=0.2593$ ) 
6. Low disability..etc...subtle cognitive deficits - The authors use' low disability' and then use GOS perhaps it would be better to stick to one standard. Also - how are subtle cognitive deficits measured - what neuro-yah examination is undertaken and when, what is the preop baseline?

Agree - we have used GOS throughout the manuscript for consistency. We have added in further details regarding psychological testing in the results.

The majority of patients had favourable outcomes and made a good recovery, with a Glasgow outcome score of 5 (42.1\% in the clipped cohort, $65.7 \%$ in the coiled) or 4 (15.8\% and $9.8 \%$ respectively). Cognitive and memory deficits, which were identified by the therapy teams at discharge and formally assessed by neuropsychology follow-up, were seen in $5.3 \%$ in the clipped group and $6.2 \%$ in the coiled.

\title{
7. At one year, a further ten patients had died or were lost to follow-up.- What are the causes of these deaths?
}

\begin{abstract}
We know the cause of death if directly related to the SAH (and were discussed with our neurosurgical unit) or if the patient died in our hospital from other causes. Unfortunately, if they died at their local hospital from unrelated conditions we don't have access to the cause of death (we know the date of death). We acknowledge this as a study limitation, and have re-worded the text to reflect this.
\end{abstract}

At one year, a further seven patients were lost to follow up and three patients had died from unrelated causes.

8. Rate of rebleeding after coiling 5.3\% - this seems higher than what is reported for ISAT. Also, there seems to be rebreeding without aneurysm recurrence too - how do the authors explain that?

5.3\% was higher than that seen in ISAT. In ISAT 28 rebleed in the first year, and 33 in subsequent follow-up (although this is not specific to ACOM aneurysms). This has now been reflected in the discussion, and we explain the late rebleed below.

Only one patient (0.88\%) had a late rebleed on day 433; this was from an aneurysm recurrence which was not picked up earlier as he did not attend his follow-up.

Our rate of rebleeding was 5.3\% following coiling (6 patients, 5 rebled early and 1 late) and 0\% following clipping at a median follow up of 3 years. The variation from ISAT (higher for the coiled cohort, lower for the clipped) (Molyneux et al. 2002, 2015) could be due to patient demographics, patient selection and our specific focus on ACOM aneurysms.

9. Discussion Alleway 2103 - should be 2013 - This is probably a top and should be corrected.

\section{Changed - thank you}

Overall, the flow of the manuscript can be better, the discussion is a bit repetitive and perhaps a flowchart detailing the numbers would help very much.

Thank you -we have tried to improve the flow by making the manuscript more succinct, particularly the discussion. We hope that it now reads better. 
Associate Editor's Comments to Author:

Associate Editor

Comments to the Author:

Please see reviewer comments - while this is of interest to the readership; needs a bit more work.

Many thanks for the opportunity to improve our manuscript. We hope it is a valuable addition to the literature. 
Figure 1; Graph to show the trend in treatment with time, following the NCEPOD audit 'Managing the flow' which mandates treatment within 48 hours, and an expansion of the neurosurgical unit (Alleway et al. 2013). CNS = clinical nurse specialist, NCEPOD = National confidential enquiry into patient outcome and death. 


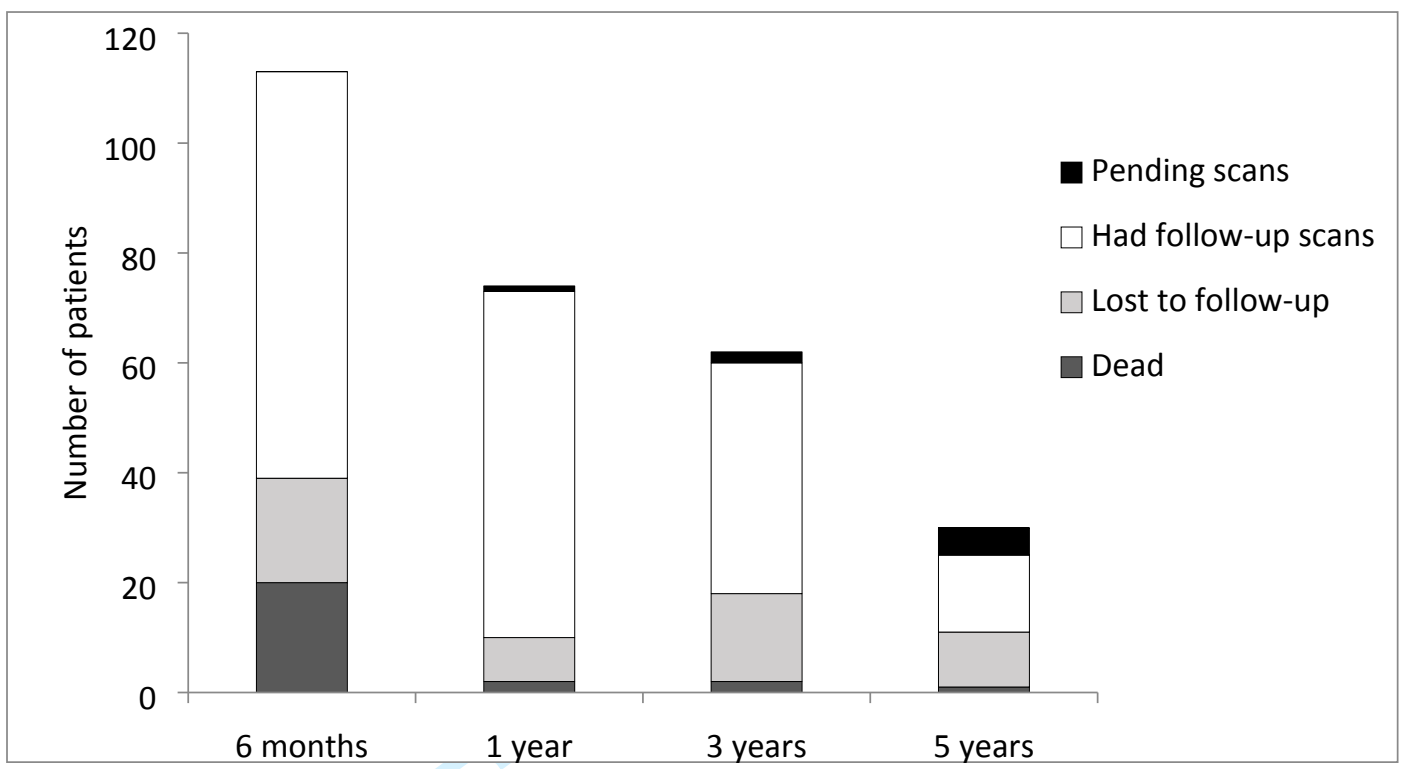

Figure 2; Graphs to show follow-up over time for coiled cohort. 


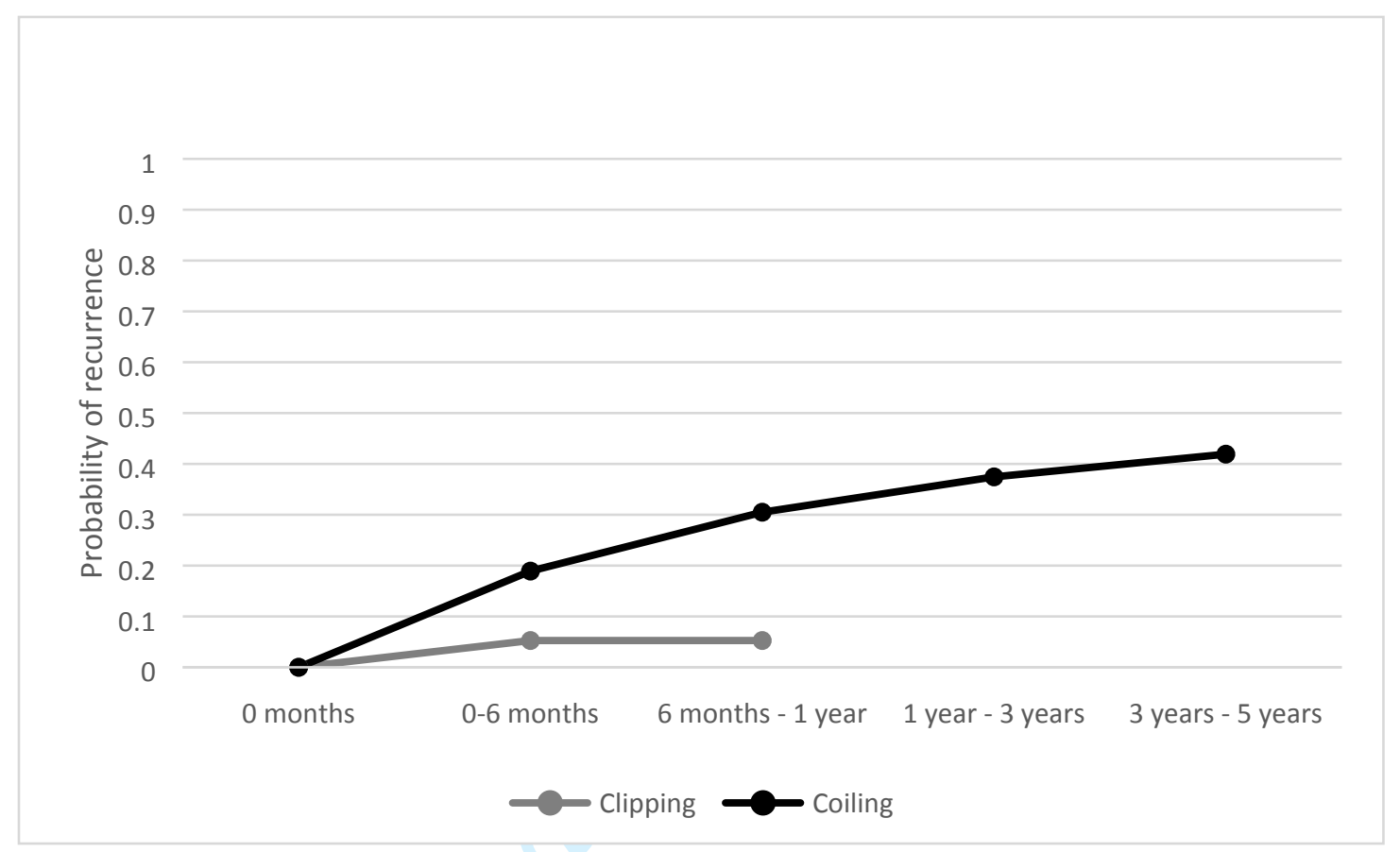

Figure 3; The probability of aneurysm recurrence over time, censored appropriately for death or lost to follow-up. 
Figure 4; Graph to show outcomes over time for the coiled cohort. It identifies patients with no recurrence, residual or new recurrence and whether the recurrent aneurysm was stable or unstable. The aneurysm was labelled as unstable if it was retreated, awaiting retreatment or, in one case, had a late rebleed. At 6 months, 74 patients had follow-up scans. 20 patients had died (14 SAH related), and 19 were lost to follow-up or transferred to their local service. At one year, a further seven patients were lost to follow-up and three had died from unrelated causes. 